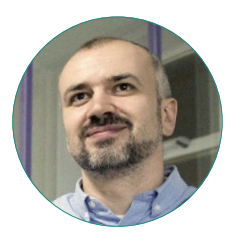

А. М. Корбут

\title{
ОДОМАШНИВАНИЕ ИСКУССТВЕННОГО ИНТЕЛЛЕКТА: УМНЫЕ КОЛОНКИ И ТРАНСФОРМАЦИЯ ПОВСЕДНЕВНОЙ ЖИЗНИ
}

\section{Правильная ссылка на статью:}

Корбут А. М. Одомашнивание искусственного интеллекта: умные колонки и трансформация повседневной жизни // Мониторинг общественного мнения: экономические и социальные перемены. 2021. № 1. С. 193-216. https://doi.org/10.14515/monitoring.2021.1.1808.

\section{For citation:}

Korbut A. M. (2021) Domestication of Artificial Intelligence: Smart Speakers and Transformation of Everyday Life. Monitoring of Public Opinion: Economic and Social Changes. No. 1. P. 193-216. https://doi.org/10.14515/monitoring.2021.1.1808. (In Russ.) 
ОДОМАШНИВАНИЕ ИСКУССТВЕННОГО ИНТЕЛЛЕКТА: УМНЫЕ КОЛОНКИ И ТРАНСФОРМАЦИЯ ПОВСЕДНЕВНОЙ ЖИЗНИ

КОРБУТ Андрей Михайлович - кандидат социологических наук, старший научный сотрудник Центра фундаментальной социологии, Национальный исследовательский университет "Высшая школа экономики", Москва, Россия E-MAIL: akorbut@hse.ru https://orcid.org/0000-0003-3835-9863

Аннотация. Появившись относительно недавно, в 2014 г., умные колонки (портативные колонки с интегрированным голосовым помощником) уже стали для многих пользователей привычной частью домашней обстановки. В данной работе рассматривается, какие трансформации происходят в социальных практиках в результате появления в домохозяйствах умных колонок. В статье анализируются два аспекта одомашнивания искусственного интеллекта при использовании умных колонок: 1) включение умных устройств в повседневную домашнюю культуру, 2) формы и способы взаимодействия с умными устройствами. Преобразование домашней социальности, к которому приводит появление умных колонок, заключается, с одной стороны, в изменении рутинных домашних практик (приготовления еды, уборки, прослушивания музыки, установки будильника, выяснения прогноза погоды и т.д.). В некоторых случаях умные колонки меняют только фон деятельности, но в других случаях трансформируют способ и частоту ее осуществления. С другой стороны, появление умных колонок приводит к новым формам коммуникации, которые ра-
DOMESTICATION OF ARTIFICIAL INTELLIGENCE: SMART SPEAKERS AND TRANSFORMATION OF EVERYDAY LIFE

Andrei M. KORBUT ${ }^{1}$ - Cand. Sci. (Soc.), Senior Researcher at the Center for Fundamental Sociology

E-MAIL: akorbut@hse.ru

https://orcid.org/0000-0003-3835-9863

\section{${ }^{1}$ National Research University Higher School of Economics, Moscow, Russia}

Abstract. Being a relatively recent invention (they appeared in 2014), smart speakers (speakers with an integrated voice assistant) have already become part of many users' home environment. This paper examines what kind of transformations occur in social practices due to the introduction of smart speakers to the domestic life. The article analyses two aspects of the 'domestication' of artificial intelligence when using smart speakers: (1) involvement of intelligent devices in everyday home culture and (2) forms and ways of interacting with smart devices. Transformation of home sociality that the introduction of smart speakers leads to, consists, on the one hand, in changing routine domestic practices (cooking, cleaning, music listening, setting an alarm clock, checking the weather report, etc.). In some cases, smart speakers only change the activity's background, but they transform the same way and frequency of activities in other cases. On the other hand, smart speakers' introduction leads to new forms of communication that were previously unusual in domestic life. For example, users increasingly shift to loud speech or avoid specific phrases and expressions to protect privacy. Relying on 
нее были непривычны для домашней жизни. Например, пользователи чаще используют громкую речь, избегают определенных фраз и выражений для сохранения приватности или начинают фразы с обращения к собеседнику.

В статье с опорой на данные существующих исследований, автоэтнографические наблюдения автора и анализ фрагментов разговоров с умной колонкой показывается, что, несмотря на стремление разработчиков умных колонок сделать их использование "естественным", подобные разговаривающие устройства требуют от пользователей не просто применения к искусственному интеллекту привычного способа взаимодействия, а выработки новых форм взаимодействия и социальности.

Ключевые слова: умные колонки, социология повседневности, дом, искусственный интеллект, этнометодология

Благодарность. Статья подготовлена в сотрудничестве с Лабораторией геймификации Сбербанка. the existing research data and his own auto-ethnographic observations and analysis of parts of conversations with the smart speaker, the author shows that, despite the inventor's desire to make the use of smart speakers 'natural', users do need to develop new forms of interaction and sociality to interact with Al.

Keywords: smart speakers, sociology of everyday life, home, artificial intelligence, ethnomethodology

Acknowledgments. The article is prepared in collaboration with the SberBank Gamification Lab. 


\section{Введение}

Сегодня многие цифровые технологии основываются на искусственном интеллекте (или машинном обучении, как предпочитают говорить специалисты, чтобы не создавать завышенных ожиданий относительно "интеллектуальности" подобных систем $\left.{ }^{1}\right)$. С социологической точки зрения искусственный интеллект интересен как элемент культурного воображаемого, то есть в контексте того, как различные социальные группы представляют себе сущность искусственного интеллекта, его возможности и ограничения, как он изображается в медиа и становится элементом дискурсивных практик (см., например, [Cave, Dihal, Dillon, 2020]). Bce это важные элементы социальных процессов, однако у искусственного интеллекта есть и более прагматическая сторона: чтобы оказывать то воздействие, которое от них ожидают разработчики, технологии искусственного интеллекта должны "оповседневниваться", рутинизироваться, становиться знакомой и привычной частью обыденных ситуаций, в которых ими будут пользоваться не только люди со специальными навыками, но и обычные пользователи, решающие рутинные профессиональные или бытовые задачи. Такое повседневное обживание технологий искусственного интеллекта происходит в обстоятельствах, где реальные практики обусловливаются структурами повседневной жизни и в меньшей степени поддаются контролю со стороны разработчиков, чем культурное воображаемое. Здесь на первый план выходит социология повседневной жизни как инструмент понимания того, каким образом люди вступают во взаимодействие с искусственным интеллектом и наделяют его смыслом.

Одомашнивание машин - давняя мечта, которая двигала и до сих пор движет разработчиками искусственного интеллекта. Хотя сегодня многие из них призывают не слишком доверять прогнозам о скором проникновении умных машин во все области человеческой жизни и даже отказаться от самой фразы "искусственный интеллект" как вводящей в заблуждение, мы по-прежнему сталкиваемся с обещаниями появления "домашних роботов", которыми сопровождается выпуск прототипов или действующих устройств и технологий, основанных на искусственном интеллекте. Некоторые из этих устройств уже покинули лаборатории и проникли в дома обычных людей. Самыми известными из них стали домашние роботы-пылесосы и умные колонки. И если роботы-пылесосы вряд ли могут считаться вершиной искусственного интеллекта, то умные колонки в полной мере соответствуют идеалу разработчиков, продавцов и поборников технологий искусственного интеллекта: это бытовые устройства, которые постоянно находятся дома, не требуют специальных навыков для пользования ими и взаимодействуют с людьми посредством естественного языка.

В данной статье мы рассмотрим умные колонки как новый элемент домашней социальности. Хотя обычные пользователи уже довольно давно сталкиваются с голосовыми помощниками (в смартфонах или компьютерах) и рекомендательными сервисами, разговаривают с телефонными чат-ботами, умные колонки вводят

\footnotetext{
1 В данной статье мы будем использовать термин "искусственный интеллект", поскольку он получил наибольшее распространение в качестве собирательного названия определенного класса технологий, но при этом нас не будет интересовать, насколько такое название справедливо и действительно ли это "интеллект". Мы изучаем взаимодействие с соответствующими технологиями, а не то, моделируют ли они особенности какого-либо "естественного" интеллекта.
} 
искусственный интеллект в парадигмально повседневную среду 一 домашнюю обстановку, и максимально упрощают взаимодействие с ним. Каким образом в этом случае происходит одомашнивание искусственного интеллекта? Как искусственный интеллект становится партнером по взаимодействию и опосредует рутинные домашние практики? Мы рассмотрим эти вопросы, отталкиваясь от ряда подходов в социологии повседневности, прежде всего - этнометодологии. Предметом нашего внимания будут исключительно бытовые практики. Мы остановимся на двух аспектах одомашнивания умных колонок - их включенности в домашнюю культуру и способах взаимодействия с ними ${ }^{2}$. При этом задача будет заключаться в выделении и первичном описании ключевых социальных феноменов, связанных с этими практиками одомашнивания. В своем анализе мы будем отталкиваться от данных, полученных в рамках других исследований, от собственного опыта пользования умной колонкой и от данных, собранных в рамках осуществляемого нами с коллегами исследования особенностей взаимодействия с умными колонками.

Но прежде чем приступить к рассмотрению особенностей одомашнивания умных колонок, остановимся на специфике социологического интереса к взаимосвязи между домом и технологиями и, в частности, умными колонками.

\section{Дом и технологии}

Дом был важным, но парадоксальным объектом изучения в социологии на всем протяжении ее истории. С одной стороны, дом делает явными многие аспекты социальной жизни, связанные с центральными вопросами социальных наук: соотношение работы и досуга [Oakley, 2018], общественное разделение труда [Phizacklea, Wolkowitz, 1995], структуру гендерных [Pink, 2004; Scicluna, 2017] и классовых [Gilbert, 2016] отношений в обществе, процессы социализации [Bartlett, 1997], хозяйственную деятельность [Хокшилд, Мачун, 2020], родственные и семейные отношения [Smart, 2007], представление себя другим [Riggins, 1991], символическую организацию памяти и идентичности [Csikszentmihalyi, Rochberg-Halton, 1981; Chevalier, 1999]. При этом дом был своеобразным идеалом, в котором воплощались как обыденные, так и исследовательские представления о совершенной жизни [Chambers, 2020]. С другой стороны, собственно дом слишком легко не замечается, становясь для социологов экраном, на который проецируются другие, скрытые для невооруженного взгляда процессы и механизмы. Например, в известном приложении «Дом, или Перевернутый мир» к своей

\footnotetext{
2 Это не означает, разумеется, что другие аспекты “оповседневнивания" умных колонок не представляют интереса. Например, важную социологическую информацию дают социально-демографические характеристики пользователей умных колонок. Согласно данным Pew Research Center, в США портрет пользователя умных колонок выглядит следующим образом: молодой (32\% пользователей в возрасте 18-29 лет) или средних лет (28\% пользователей в возрасте 30-49 лет), со средним и высоким доходом, с высшим образованием, житель города или пригорода. См.: Auxier B. 5 Things to Know about Americans and Their Smart Speakers. URL: https://www.pewresearch.org/facttank/2019/11/21/5-things-to-know-about-americans-and-their-smart-speakers/ (дата обращения: 17.01.2021).

Данные о российских пользователях умных колонок гораздо скуднее. Самое общее представление можно получить, например, из аналитического отчета компании Just Al. См.: Рынок умных колонок и голосовых ассистентов: Россия и мир. Прогнозы и тренды // Just Al. 2020. 7 сентября. URL: https://just-ai.com/blog/rynok-umnyh-kolonok-i-golosovyhassistentov-rossiya-i-mir-prognozy-i-trendy (дата обращения: 17.01.2021).

Однако в любом случае в данной статье мы будем рассматривать только те изменения, которые умные колонки привносят в домашнюю социальность в качестве специфического (разговаривающего) объекта. Проблема пользователя, его характеристик и статуса требует отдельного обсуждения.
} 
книге "Практический смысл" Пьер Бурдье рассматривает дом как "микрокосм, организованный в соответствии с теми же оппозициями, которые организуют универсум" [Бурдье, 2001: 528]. И даже если дом противопоставляется окружающему миру как особая область порядка, он все равно отражает этот мир, пусть и в перевернутом виде.

Социологи, не рассматривавшие дом как проекцию социальных кодов и практик, обращали внимание на то, что он представляет собой особое пространство повседневной жизни, где осуществляются практики, которые сложно или невозможно встретить в других местах [Pink et al., 2017]. В первую очередь это практики социальных взаимоотношений (интимных, родственных, дружеских; их принято относить к области "приватного", в противоположность "публичному"), но также - что важнее для нашего исследования,-технологические практики. Дом аккумулирует в себе определенные формы деятельности, а последние всегда имеют специфическое материальное, в том числе технологическое воплощение. Домашние практики заставляют инженеров и дизайнеров находить новые технические решения для их осуществления (например, разогрев еды и стирка белья радикально изменились в результате появления микроволновой печи и стиральной машины), но также сами оказываются производными от новых технологий, проникающих в дома и создающих соответствующие практики. Эта связь между домом и технологиями зачастую ускользает от внимания исследователей. Поставленный Дебби Хиндус в 1999 г. диагноз (“...технологии в домах получают мало внимания в исследовательском сообществе» [Hindus, 1999: 199]) остается справедливым.

Цифровые технологии, разумеется, не обошли дом стороной. Когда начали появляться вычислительные машины, способные решать не только узкоспециализированные задачи, многие увидели их потенциал для радикального преображения домашней жизни. Наиболее полное воплощение эти ожидания получили в идее умного дома, которая до сих привлекает как разработчиков, так и обычных пользователей. Согласно определению Фрэнсис Олдрич, умный дом - это “жилище, оснащенное вычислительно-информационными технологиями, которые предвосхищают и реагируют на потребности жильцов, обеспечивая им комфорт, удобство, безопасность и развлечение посредством управления технологиями внутри дома и связи с внешним миром" [Aldrich, 2003: 17]. И хотя воплощение этой мечты оказалось не таким простым, как представлялось первоначально, различные элементы умного дома, включая умные термостаты, умные лампочки, умные замки, умные жалюзи, умные чайники, умные холодильники и, конечно, умные колонки, уже с нами. Умный дом по своей природе социален, поскольку им следует управлять и он должен "реагировать на потребности жильцов", прежде всего через голосовые интерфейсы. До появления голосовых помощников это было почти невозможно. Сегодня вычислительные мощности и, главное, достижения в машинном обучении позволяют вести с различными устройствами полноценный (в техническом смысле) разговор - пользователи не просто дают голосовые команды, но обмениваются с "умными" устройствами репликами. В этом смысле современные умные устройства меняют социальность дома: в домашнем пространстве появляется еще один социальный объект, с которым можно разгова- 
ривать и который при этом обладает рядом тревожных социальных способностей вроде способности постоянно слышать то, что произносится в доме.

Эта видоизмененная домашняя социальность требует социологического анализа, удобный объект для которого - использование умных колонок как распространенного домашнего устройства с голосовым интерфейсом ${ }^{3}$. Этот анализ особенно важен в ситуации, когда пандемия COVID-19 может существенно повлиять на формы организации домашней жизни и сместить или даже стереть границу между домом и работой.

\section{Умная колонка как элемент домашней социальности}

Дом - не только пространство, в котором отражается представление людей о том, кто они такие и какое положение в обществе они занимают, и которое играет принципиально важную роль в проведении границы между приватным и публичным. Дом - это также пространство повседневной жизни, где люди осуществляют специфические практики, связанные с организацией пространства и времени, управлением материальной средой, телесностью и коммуникацией с другими. Появление умных колонок в домах в той или иной степени затронуло все эти аспекты домашней социальности. У людей (что немаловажно, не обязательно владеющих смартфоном или компьютером) появился не только новый партнер по взаимодействию, но и новый инструмент осуществления повседневных практик, который способен менять традиционные формы домашней активности, а также предлагать новые возможности. Для социологии это важная и интересная ситуация, позволяющая наблюдать, с одной стороны, как происходит проникновение в повседневность новой технологии и с другой - как осуществляется одомашнивание искусственного интеллекта в той его форме, которая наиболее понятна, привычна и доступна, в форме разговорного искусственного интеллекта.

Основная характеристика умной колонки как домашнего устройства, разумеется,- возможность коммуникации с ней посредством голоса. Однако эта коммуникация предполагает ряд дополнительных условий, определяемых как техническими характеристиками устройства, так и особенностями среды, в которой оно должно использоваться.

Во-первых, умная колонка должна быть фоновым объектом, который всегда под рукой. Колонка должна быть «in hand", подручной (если воспользоваться термином М. Хайдеггера), и встраиваться в совокупность других домашних объектов. В отличие от, например, телевизора, который обычно предполагает длительное фокусирование на нем пользователя или пользователей (даже если телевизор работает в фоновом режиме), колонка должна быть малозаметной и только время от времени становится объектом внимания.

\footnotetext{
3 В 2019 г. по всему миру было продано 146,9 млн умных колонок. См.: Koksal I. The Sales of Smart Speakers Skyrocketed // Forbes. 2020. 10 March. URL: https://www.forbes.com/sites/ilkerkoksal/2020/03/10/the-sales-ofsmart-speakers-skyrocketed/?sh=6788f07538ae (дата обращения: 17.01.2021).

В России, по данным “М. Видео-Эльдорадо", в первые семь месяцев 2020 г. было продано 1,3 млн устройств портативной акустики и умных колонок (причем продажи умных колонок выросли в 6,5 раз по сравнению с тем же периодом прошлого года). См.: Аналитика "М. Видео-Эльдорадо": продажи умных колонок в России в 2020 году выросли в 7 раз // CNews. 2020. 16 сентября. URL: https://cnews.ru/link/n515185 (дата обращения: 17.01.2021).
} 
Во-вторых, умная колонка должна располагаться в доме так, чтобы она всегда была доступна для голоса. Поскольку умная колонка должна быть присоединена к источнику питания, что сильно ограничивает возможности ее перемещения внутри дома, с учетом конструкции современных жилищ это означает, что пользователям надо либо покупать несколько колонок, либо выбирать, в каком месте дома колонка наиболее нужна. Выбор места, в свою очередь, будет связан с тем, как колонка используется, поскольку любой дом представляет собой совокупность практических пространств, в которых люди осуществляют определенные виды деятельности (см. об этом ниже).

В-третьих, умная колонка должна служить домашним помощником. Это связано как с функционалом умной колонки (она может озвучить прогноз погоды, запустить музыку, сообщить нужный адрес, подсказать перевод или значение слова, рассказать анекдот и т.д.), так и с тем, что колонка представляет собой, по сути, физический интерфейс для голосового помощника (Alexa, Siri, Алисы или Мapyси). В доме умная колонка исполняет служебную роль. Это выражается, среди прочего, в том, что она говорит, только когда к ней обращаются.

В-четвертых, так как умная колонка - домашнее устройство, она оказывается предметом коллективного пользования. Дом - это чаще всего место группового проживания, но даже в случае одиноко живущих людей колонка может быть объектом групповых действий (например, при посещении гостей). Эта особенность стимулируется определенными функциями, которые закладывают в колонку производители. Например, Amazon Echo, самая популярная в мире умная колонка, имеет функцию "Announcements", которая позволяет делать "объявления" для всего дома, то есть создавать систему “внутренней связи" (при наличии нескольких установленных колонок).

Эти социальные особенности использования умных колонок не остались без внимания ученых. Несмотря на новизну данной технологии, в ряде работ были выявлены важные особенности повседневного использования умных колонок в домах. Например, Э. Бенето и ее коллеги из Вашингтонского университета исследовали десять семей, которые в течение месяца впервые пользовались умной колонкой Amazon Echo Dot ${ }^{4}$ [Beneteau et al., 2019, 2020a, 2020b]. Авторов прежде всего интересовал вопрос внедрения и распространения инноваций. Они выяснили, что пользователи постепенно сокращают количество сценариев использования колонки и задействуют только небольшой набор ее функций, что связано с тем, как они выявляют функциональность колонки. Чаще всего они спрашивали саму колонку о ее возможностях (хотя с течением времени отказывались от этого), использовали метод проб и ошибок или получали советы от родственников, друзей и знакомых и в гораздо меньшей степени полагались на информацию, предоставляемую производителем. Это исследование показывает, что, попадая в домашнюю среду, колонка становится элементом доминирующих там форм социальности, включая формы обучения и передачи знаний. Если предлагаемые колонкой формы социальности не соответствуют домашним, это существенно снижает ее возможности в качестве партнера по взаимодействию.

\footnotetext{
${ }^{4}$ Amazon Echo Dot-более портативная версия умной колонки Amazon Echo. В отличие от Echo, Echo Dot имеeт небольшие размеры и лишена отдельного дисплея.
} 
Более интеракционный ракурс был выбран в исследовании М. Поршерона и его коллег [Porcheron et al., 2018; Fischer et al., 2019; Reeves, 2017]. С помощью конверсационного анализа они проанализировали взаимодействия пяти семей с умной колонкой Amazon Echo Dot в течение месяца. Их интересовало, каким образом взаимодействие с умной колонкой встраивается в другие домашние занятия вроде семейного ужина. Они показывают, что взаимодействие с колонкой часто разрушает параллельные формы деятельности, поскольку переключает внимание на само это взаимодействие, требующее обширных согласованных усилий членов семьи, которые при решении возникающих проблем руководствуются «политикой дома" - рутинными, устоявшимися способами домашнего разделения труда, прав и обязанностей.

Ф. Бентли и соавторы [Bentley et al., 2018] дополнили этот интеракционный анализ лонгитюдными количественными данными использования умных колонок в 88 домах в течение 110 дней. Они демонстрируют, что обращение к умным колонкам обусловливается суточными и недельными ритмами домашней жизни, связанными с рабочими графиками членов семьи и принятыми в доме формами досуга. Эта матрица домашней жизни настолько устойчива, что с течением времени способы использования умных колонок мало трансформируются. После первоначального периода тестирования возможностей колонки пользователи создают небольшой набор домашних рутин, в рамках которых эти колонки становятся необходимым элементом. Один из выводов из исследования Бентли и его коллег заключается в том, что использование умных колонок принципиально отличается от использования голосовых ассистентов, доступных на смартфонах через мобильные приложения. Умные колонки попадают в особую обстановку домашней жизни, материальная и социальная организация которой как стимулирует взаимодействие с умными колонками (ими пользуются гораздо чаще, чем голосовыми помощниками), так и создает препятствия для этого (например, содержание и форма взаимодействия с умной колонкой в значительной мере определяются местоположением колонки внутри дома).

Указанные исследования позволяют не только ответить на ряд эмпирических вопросов, касающихся особенностей использования умных колонок и их интеграции в домашнюю повседневность, но и поставить некоторые концептуальные вопросы, касающиеся отношений между пользователями подобного рода устройств и объектами, с которыми они взаимодействуют. Как отмечают М. Алач и соавторы, повсеместное распространение голосовых разговорных интерфейсов приводит к появлению “гиперсоциализированного мира, в котором все является социальным, и мы живем в кухнях, где с нами разговаривают даже тостеры" [Alač et al., 2020: 4]. Однако в основе технологий, обеспечивающих такую гиперсоциализацию, лежит представление об “индивидуальных Я как элементарных единицах социального" [ibidem]. Концентрация разработчиков на "пользователе" стабилизирует эти Я, в результате чего им приписываются устойчивые характеристики, ожидание которых закладывается в компьютерную системy [Grint, Woolgar 1997]. В результате «пользовательский опыт" не только ставится в центр процесса создания голосовых интерфейсов, но и направляет внимание исследователей. Например, М. Поршерон с соавторами, аргументи- 
руя необходимость отказа от используемого в индустрии термина "разговорные агенты" в пользу термина "голосовой пользовательский интерфейс", говорят, что "разговорное взаимодействие - ошибочное название для подобного типа человеко-компьютерной интеракции, поскольку оно смешивает взаимодействие с устройством в рамках разговора с самим разговором" [Porcheron et al., 2018: 9]. Иными словами, по мнению авторов, умная колонка, взаимодействие с которой они изучали, - это лишь то, что используется в разговоре, но не является партнером по взаимодействию, а значит, "двигателем" этой разговорной системы выступает пользователь, пытающийся решать с помощью соответствующего устройства свои практические задачи. М. Алач и ее коллеги предлагают отказаться от такой индивидуализированной концепции пользователя в пользу представления о разговаривающих устройствах как «элементах мира, к которым мы интеракционно открыты (а не орудиях, превращающих людей в более могущественных когнитивных агентов)" [Alač et al., 2020: 9].

В данной статье мы рассмотрим вторую составляющую интеракционной открытости, о которой говорят М. Алач и соавторы. Если они предлагают переопределить субъекта, взаимодействующего с разговорным искусственным интеллектом, то мы предлагаем проанализировать, какого рода объектом является разговаривающее устройство, в частности, умная колонка. Вопрос понимания объектов, с которыми взаимодействуют люди,- один из самых обсуждаемых в современной социологии. После долгого периода игнорирования роли объектов в социальном мире либо рассмотрения их как смысловых субстратов, меняющихся в зависимости от того, какой смысл им приписывают люди ${ }^{5}$, ряд исследователей предложили радикально "пересобрать" социальное, чтобы за объектами или вещами была признана исполняемая ими конститутивная роль [Ерофеева, 2017; Латур, 2014; Molotch, 2011]. Этот социологический "объективизм", однако, не позволяет понять, каков интеракционный статус объектов в социальном мире, который радикально меняется, когда объекты сами начинают разговаривать с людьми. Для этого нужны другие понятия и подходы, которые мы предложим ниже. В этом отношении мы продолжаем линию анализа "объектов как практических достижений" [Nevile et al., 2014: 4], то есть того, как объекты возникают во взаимодействии, но при этом не конструируются пользователями. Мы распространим эту логику анализа на специфические объекты - разговаривающие машины. На наш взгляд, они позволяют поставить ряд вопросов, которые были невозможны до появления этих объектов в повседневных ситуациях.

Указанные выше исследования дают возможность выделить два ключевых аспекта взаимодействия с умными колонками: их интеграцию в домашнюю повседневную культуру и собственно коммуникацию с ними.

\section{Умные колонки и привычные домашние практики}

Дом - специфическое социальное пространство. Его специфика определяется не только тем, что дом в определенном смысле противостоит рабочему месту как пространство досуга (хотя в последние годы это меняется и сегодня дом все чаще

\footnotetext{
5 Наиболее последовательный теоретик такого подхода, вероятно, Герберт Блумер [Блумер, 2017].
} 
становится местом работы [Oakley, 2018]), но и тем, что дом - это пространство приватное, то есть такое, доступ к которому регулируется особыми социальными правилами (как юридическими, так и неформальными) и которое выступает своеобразным продолжением человека: его/ее личности, идентичности и даже телесности. С точки зрения социологии повседневности дом обладает следующими характеристиками:

1) Дом - это опривыченное пространство. Длительное нахождение в доме, его относительно небольшая территория и стабильный набор действий, совершаемых в рамках ежедневных и еженедельных рутин, приводят к формированию ряда привычек, связанных как с перемещениями внутри дома, так и с формами активности в нем. Например, есть ряд привычных действий, которые совершаются людьми дома после пробуждения или перед тем, как лечь спать. Эти привычки запечатлеваются в теле настолько глубоко, что мы можем перемещаться внутри дома и осуществлять различные действия, уделяя этому гораздо меньше внимания, чем в любой другой обстановке. Мы можем попасть в нужное место дома или найти нужную вещь с закрытыми глазами.

2) Чтобы эти привычки работали, необходимо определенным образом преобразовать домашнюю среду: она должна стать "слепком" домашних действий. В результате регулярного совершения определенных действий вещи внутри дома распределяются так, что какие-то из них оказываются на периферии привычных практик, а какие-то - всегда под рукой. В этом смысле пространство дома - это аккумулированное пространство. Вещи в нем "оседают" в различных местах в зависимости от того, в какие практики они включены. Одни вещи оказываются у задней стенки шкафа на верхней полке, другие - на раковине в ванной. Появление новых вещей порождает своеобразные "волны" в этом аккумулированном пространстве, заставляя переконфигурировать сложившиеся кластеры вещей. (Эти "волны" могут быть как очень небольшими, например, когда мы немного меняем положение продуктов в холодильнике, чтобы добавить туда пакет молока, так и довольно значительными, например, когда нам приходится перебирать кладовку, чтобы положить туда чемодан.) И практически в любом доме существуют также рутинные способы избавления от вещей (например, с помощью ведра для мусора). При этом аккумуляция определяется не только частотой использования тех или иных вещей, но и структурой самого пространства, вмещающего только вещи определенного размера, и локальной историей дома (например, тем, что в доме могут в разное время проживать разные люди, в результате действий которых те или иные вещи оказываются в тех или иных местах).

3) Еще одна важная характеристика дома, тесно связанная с двумя предыдущими, - зональность. В любом доме есть различные пространства действия (или "центры активности" [Crabtree, 2003: 12]), которые различаются между собой особой материальной конфигурацией, отражающей и обеспечивающей совершаемые там практики. Отчасти эта зональность определяется инфраструктурой жилища (в нем есть "кухня", "туалет", "ванная", "спальня" и т.д.), но в любом случае эта инфраструктура должна быть включена в привычные домашние практики, чтобы можно было говорить о ее влиянии. "Кухню” делает 
"кухней" не то, как спроектирован дом, а то, используют ли жильцы это пространство в качестве кухни. При этом зоны внутри дома имеют пространственно и темпорально подвижные границы. Например, потребление еды или чтение могут происходить в разных местах. В этом смысле зоны внутри дома - скорее полюса притяжения, а не выделенные территории для определенных практик. Тем не менее можно говорить, что в определенных местах конкретные формы деятельности совершаются чаще. Например, телевизор чаще располагается в гостиной, чем в других местах дома, и, соответственно, связанные с телевизором индивидуальные и коллективные практики чаще осуществляются именно там.

Что происходит, когда в это опривыченное, аккумулированное, зонированное пространство дома попадает умная колонка? Оказывает ли ее появление одинаковое воздействие на три выделенных аспекта дома или какие-то из них трансформируются сильнее? Чтобы ответить на эти вопросы, необходимо внимательнее посмотреть на то, что именно меняется в привычных домашних практиках после появления умной колонки.

Во-первых, меняется фон некоторых практик. Все действия внутри дома имеют определенный материальный фон - это может быть как некоторая конфигурация вещей, так и звуковой фон. Часто этот фон управляется самими акторами. Поскольку умная колонка предназначена для воспроизведения звуков, она меняет звуковой фон: например, определенные действия вроде приготовления еды начинают сопровождаться музыкой, проигрываемой с помощью колонки. Однако проигрывание музыки - не единственный способ, которым умные колонки влияют на фон деятельности. Во многих случаях умная колонка представляет собой фоновый источник информации, к которому периодически обращаются при осуществлении какой-то другой деятельности. Скажем, за семейным ужином участники могут задавать колонке вопросы, касающиеся значения слов или сведений по определенной теме. Такое фоновое информационное присутствие умной колонки имеет и обратную сторону - поскольку умная колонка не только сообщает, но и собирает информацию, она является постоянным фоновым слушателем всего, что говорят люди дома, причем слушателем, который подключен к интернету и может отправлять услышанное за пределы дома. У многих пользователей такое фоновое присутствие умной колонки вызывает беспокойство по поводу сохранения приватности происходящего в доме. Результатом этого беспокойства может быть как появление определенных практик пользования колонкой (например, выключение ее в определенные моменты), так и избегание тем, которые считаются пользователями непубличными.

Во-вторых, присутствие умной колонки может менять само содержание некоторых практик. В качестве примера можно привести прием гостей. Колонка может становиться не только фоновым проигрывателем музыки, но и инструментом развлечения: хозяева демонстрируют гостям, что умеет и не умеет делать искусственный интеллект, а гости устраивают "тесты", задавая колонке сложные вопросы или давая веселые задания. В этом случае застольные или дружеские практики частично меняют свое содержание, и среди знакомых практик обмена новостями, рассказывания шуток и т.д. появляется еще одна неосуществимая 
без умной колонки и использующая как ее возможности, так и ее ограничения в качестве ресурсов. При этом коммуникативные сбои в общении с колонкой могут становиться поводом для развлечения и веселья, а не раздражения и недовольства.

В-третьих, умные колонки могут менять частоту осуществления ряда привычных практик. Например, пользователи могут начать чаще пользоваться таймером, узнавать прогноз погоды и новости или прослушивать музыку, чем это было раньше. Отчасти увеличение частоты этих практик связано с ускорением процесса: получить информацию о погоде у искусственного интеллекта быстрее, чем взять подходящее устройство и запустить специальную программу или открыть веб-сайт. Но изменение частоты также связано с тем, что сама умная колонка по мере ее использования начинает ассоциироваться с определенными практиками, которые можно с ее помощью осуществлять, и эти практики могут "открепляться" от других материальных объектов (вроде смартфонов) при их осуществлении дома.

Выделенные особенности трансформации повседневных домашних практик, вызываемые появлением в доме умных колонок, позволяют утверждать, что они оказывают неодинаковое влияние на три указанных выше аспекта домашней жизни. Наиболее заметно они влияют на устоявшиеся практики, меняя их содержание или фон, а также приводя к формированию новых домашних привычек. Кумулятивность домашнего пространства умные колонки затрагивают минимально или вообще не влияют на нее, поскольку умные колонки включены скорее в коммуникативные домашние практики и лишь косвенно связаны с организацией материальной среды в доме. Что касается зональности дома, то умные колонки здесь оказывают определенное воздействие, но не такое сильное, как в случае привычных практик. Хотя есть определенные технические обстоятельства, определяющие, в каком месте дома будет находиться колонка,- например, расположение розеток, длина провода, близость стен (если звук в колонке регулируется датчиком движения, колонку нельзя ставить близко к стенам), - с социологической точки зрения важны, прежде всего, практические социальные обстоятельства, обусловливающие выбор места. Во-первых, умная колонка должна быть размещена в том месте, которое кажется жильцам наиболее подходящим, в зависимости от того, как они используют колонку и как они воспринимают ее возможности. Если колонка используется для проигрывания фоновой музыки, она может оказаться, например, на кухне. Если она используется для получения общей информации и игр, она будет в гостиной. Если важна возможность ставить будильник, колонка будет расположена в спальне. Во-вторых, колонка должна быть размещена так, чтобы она была доступна для голоса, но при этом не мешала осуществлять другие действия. Чаще всего колонки оказываются на периферии жизненного пространства той комнаты, в которой они устанавливаются.

Например, у автора настоящей статьи умная колонка после нескольких попыток "закрепления" на кухне оказалась в основной комнате, исполняющей одновременно роль спальни и гостиной (см. рис. 1). 


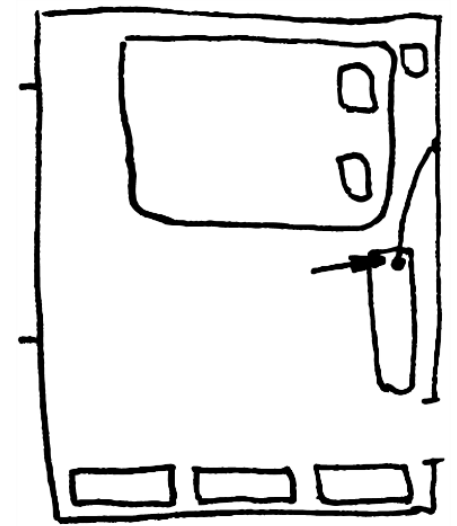

Рис. 1. Расположение умной колонки в доме автора

Такое ее расположение (на невысокой тумбе возле кровати) связано в первую очередь с доступностью для голоса людей, использующих эту комнату, и со спецификой пользования колонкой: чаще всего она сообщает информацию о погоде. В то же время колонка находится далеко от наиболее часто используемых мест в комнате: она не создает помех для потоков вещей и действий, не связанных с ней, и необходимость ее перемещения возникает только в процессе уборки.

Одомашнивание искусственного интеллекта, воплощенного в умной колонке, не только предполагает выработку особых форм коммуникации с ним и приводит к трансформации повседневных практик в доме, но и порождает новые практики, которые могут менять привычный прагматический ландшафт дома. До появления умной колонки граница между приватным и публичным проходила по границе дома, которая представляла собой, по выражению Эрвина Гофмана, своеобразную "мембрану" [Goffman, 1961: 65-66]. Эта мембрана не была абсолютно непроницаемой (у большинства домов есть двери и окна, и обеспечить их полную свето- и звукоизоляцию невозможно), но существенно ограничивала возможности перемещения людей, вещей, информации и звуков через нее. Появление умной колонки меняет эту конфигурацию приватного/публичного. Теперь граница между тем и другим проходит внутри дома и в пространстве коммуникации. Несмотря на то что все производители умных колонок заверяют покупателей, в гарантированном сохранении приватности данных, наличие в доме устройства, которое постоянно слушает разговоры, может существенно влиять на то, что и как говорится в доме. Люди могут избегать конфиденциальных разговоров в присутствии колонки или стараться не разглашать чувствительную информацию, например, отказываясь совершать покупки через умные колонки [Lau, Zimmerman, Schaub 2018; Malkin et al., 2019; Voit et al., 2020]. Отчасти такое избегающее поведение обусловливается тем, что при взаимодействии с умной колонкой посредством голоса пользователи неизбежно должны полагаться на привычное чередование ролей слушающего и говорящего, лежащее в основе любой коммуникации при помощи естественного языка. Поэтому пользователи знают, что умная колонка в принципе способна быть слушателем. Другая причина заключается в том, что 
сам факт постоянного присутствия колонки в доме и ее готовности к коммуникации приводит к приписыванию ей "подслушивающей» позиции. Если раньше приватность регулировалась физической мембраной границы дома, то теперь она регулируется доступностью или недоступностью высказываний для искусственного интеллекта.

Однако соображения приватности - не единственное обстоятельство, которое обусловливает появление новых практик внутри дома под влиянием умных колонок. Другая причина связана с коллективным использованием умных колонок. У колонки может быть несколько пользователей, конкурирующих между собой за управление ею. Колонка провоцирует такую конкуренцию, делая всех пользователей равнозначными. Это может приводить к тому, что пользователи иногда управляют не столько действиями колонки, сколько действиями других пользователей в отношении колонки (например, корректируют чужие запросы) [Porcheron et al., 2018].

Еще один аспект одомашнивания искусственного интеллекта - возможность с ним "играть". Здесь уместно воспользоваться социологическим различением ситуативной игры (play) и формальной игры (game) [Mead, 2015]. С умными колонками возможны обе, но в данном случае они имеют свою специфику, что позволяет говорить о них как новых практиках, связанных с появлением умных колонок в домах. Что касается ситуативной игры, то, конечно, умные колонки - не единственный объект, с которым осуществляется такой тип игр. Взрослые, например, играют в ситуативные игры с детьми, домашними животными и друг с другом. Однако разные типы ситуативных игр предполагают разные коммуникативные способности партнеров по игре. Поэтому в той мере, в какой о разговорном искусственном интеллекте можно говорить как о партнере по взаимодействию, его коммуникативные особенности будут требовать особых типов ситуативных игр. Например, пользователи могут пытаться заставить искусственный интеллект произнести определенное слово в ситуации, когда он запрограммирован не исполнять просьбу типа "X, скажи Y» (в собранных нами данных есть несколько таких ситуаций). Эти же коммуникативные особенности искусственного интеллекта могут использоваться в формальных играх, где пользователи должны выполнять определенные действия, предусмотренные правилами. Так, некоторые из подобных игр предполагают, что пользовать должен с помощью одной фразы заставить умную колонку произнести определенное слово, не называя это слово или его производные.

\section{Коммуникация с умной колонкой}

Главное, с чем приходится сталкиваться пользователю умной колонки,- - это коммуникативные особенности разговорного искусственного интеллекта как партнера по взаимодействию. Во многом процесс одомашнивания заключается в том, что пользователи приспосабливаются к тому, как искусственный партнер говорит, делает паузы, отвечает на вопросы, задает вопросы и т. д. Несмотря на значительный прогресс, достигнутый в последние годы, разговорный искусственный интеллект все еще демонстрирует способности, не позволяющие вести с ним "нормальный" разговор и приводящие к многочисленным сбоям во взаимодей- 
ствии (хотя, как указывалось выше, эти сбои могут быть не только источником недовольства, но и поводом для развлечения).

В данном случае отправной точкой в анализе коммуникации с разговорным искусственным интеллектом лучше брать не дихотомическую шкалу “человеческая коммуникация - все другие формы коммуникации", поскольку и сама человеческая коммуникация осуществляется в очень разнообразных формах, и коммуникация с другими партнерами по взаимодействию не всегда оценивается с точки зрения того, является ли собеседник человеком. Лучше исходить из того, что существует коммуникативный спектр, в котором разговорный искусственный интеллект занимает свое место (или даже несколько мест).

Взаимодействие с умной колонкой разворачивается как последовательность высказываний, в которой действуют стандартные ожидания относительно диалога: что реплики будут сменять друг друга без длинных пауз между ними и что собеседники не будут говорить одновременно [Сакс, Шеглофф, Джефферсон, 2015]. При этом в каждой своей ответной реплике говорящий должен демонстрировать понимание сказанного ранее и делать уместные высказывания. Современные умные колонки с трудом могут соблюдать эти условия, что побуждает пользователей находить способы корректировки своих действий и действий колонки. Наиболее распространенные способы исправления возникающих проблем таковы:

- просодическая модификация (повышение громкости, замедление речи, интонационные подчеркивания);

- семантическая модификация (например, замена слов и сокращение длины фраз);

- фонетическая модификация (например, пользователи замолкают после фразы, чтобы колонке было легче распознать сказанное).

В рамках диалога с умной колонкой важное значение имеет то, как пользователи интерпретируют действия искусственного интеллекта. В этом отношении важны два момента:

1) Молчание колонки. Поскольку взаимодействие с колонкой ведется в разговорном формате, для пользователей любое молчание со стороны колонки приобретает смысл в связи с текущей ситуацией и чаще всего интерпретируется либо как время, когда колонка "думает" (то есть совершает какие-то операции), либо как свидетельство проблемы, с которой столкнулась колонка.

В качестве примера ориентации на молчание как "значащее" действие в разговоре с умной колонкой можно привести следующий эпизод ${ }^{6}$ :

40 П1 х:::: а: какой тебе нравится фильм

41 (.) больше всего?

$42 \quad(4.3)$

43 П2 (всё сломалась)

\footnotetext{
${ }^{6}$ В нижеследующих транскриптах применяется конверс-аналитическая система транскрибирования речи, разработанная Гейл Джефферсон. Используются следующие символы: П-пользователь, А-Алиса, (0.0)-паузы в целых и десятых долях секунды, (.) - пауза около 0,1 секунды, :::-растягивания звука (количество двоеточий отражает продолжительность растягивания), слово - акцентирование, $\uparrow \downarrow-$ повышение и понижение тона, * * понижение громкости в выделенном фрагменте, [-момент наложения реплик, (слово) - неуверенное распознавание, <>- более медленное произнесение. Все записи, транскрипты которых приводятся ниже, были собраны автором и командой исследователей, изучающих взаимодействие пользователей с умными колонками.
} 
44

$$
\text { (0.5) }
$$

45 П1 алло алиса привет ты здесь

Длительное молчание голосового помощника (строка 42) после прямого вопроса создает у пользователя 2 впечатление, что помощник "сломалась". Термин "сломалась" может означать разные вещи, однако реакция пользователя 1 в строке 44 показывает, что для него проблема заключается не в физической поломке, а в коммуникативном сбое: Алиса перестала разговаривать и ее надо "расшевелить", для чего применяются типичные "стартовые" выражения, которые чаще всего используются для начала разговора или начала фразы ("алло", "привет»). В этом случае молчание колонки расценивается пользователями как свидетельство возникшей проблемы, и эта проблема заключается не в особенностях формулировки вопроса, а в выпадении голосового помощника из взаимодействия.

2) Ответы колонки представляют собой ресурс для ведения диалога и выявления проблем: для пользователя все действия колонки являются индикатором того, что происходит сейчас, происходило ранее и может происходить далее в разговоре. Но иногда информация, которую пользователи извлекают из действий голосового помощника, не соответствует действительному положению дел, и пользователи могут видеть проблему там, где ее нет, либо не замечать существующую проблему. Например, в следующем фрагменте из другого разговора пользователь «пропускает" неуместный ответ голосового помощника:

$\begin{array}{lll}65 & \text { П } & \text { ну давай анекдот какойнибудь расскажи } \\ 66 & & (3.6) \\ 67 & \text { А } & \text { в недрах тундры (.) выдра в гетрах (.) } \\ 68 & & \text { тырит в ведра (.) ядра кедров. } \\ 69 & & (1.4) \\ 70 & \text { П } & \text { *поняно* это хто написал такой стишок? } \\ 71 & & (4.9) \\ 72 & \text { А } & \text { на сол точка ру (.) есть такой ответ (0.4) } \\ 73 & & \text { стихи пишутся о главном (.) о любви, } \\ 74 & & \text { дружбе, родине, людях, детях. } \\ 75 & & \text { (1.4) } \\ 76 & \text { П } & \text { всё ясно (.) *м* какая твоя любимая } \\ 77 & & \text { песня битлз? }\end{array}$

"Ответ" Алисы на вопрос, заданный в строке 70, не соответствует этому вопросу. Несмотря на разрушение связности разговора, пользователь реагируют подтверждающей фразой "все ясно" и задает следующий вопрос. В этом случае резкая смена темы (от "стишка" к "песням Битлз") может означать, что пользователь решил не обращать внимание на неуместность ответа Алисы, но независимо от того, осознает он этот сбой в связности разговора или нет, в самом взаимодействии проблема игнорируется.

Важный аспект коммуникативных способностей разговорного искусственного интеллекта - контекстуальная нечувствительность, которая проявляется как в ходе самого разговора, так и в предшествующих ему обстоятельствах. Во время разговора быстро обнаруживается, что умная колонка не может отслеживать локальную историю разговора, и поэтому каждую новую реплику лучше формули- 
ровать так, как если бы она была первой репликой и ей не предшествовали другие. Для пользователей это всегда непростая задача, поскольку в обычной коммуникации мы полагаемся на предшествующие реплики как на ресурс понимания текущей реплики, доступный всем участникам разговора. О том, что пользователи ориентируются на контекстуальную нечувствительность голосовых помощников, свидетельствует частое использование активирующих фраз в начале реплик. Все современные умные колонки предполагают использование активирующей фразы для начала разговора. Это может быть словосочетание («Окей, Гугл») или имя ("Алиса"). Однако пользователи часто используют активирующую фразу не только в начале разговора, но и в начале отдельных реплик внутри разговора. Как показывает следующий фрагмент, далеко не всегда это связано с технической необходимостью "разбудить" колонку.

$\begin{array}{lll}15 & \text { П } & \text { Алиса скажи (0.3) <как> (0.6) } \\ 16 & & \text { по: таджицки будет школа. } \\ 17 & & (2.2) \\ 18 & \text { А } & \text { школа на таджикском }(.) \text { ма кта: (б)е } \\ 19 & & (1.0) \\ 20 & \text { П } & \text { ф пасиба Алиса } \\ 21 & & (1.9) \\ 22 & \text { А } & \text { я стараюсь } \\ 23 & \text { П } & \uparrow \text { Алиса а какая твоя любимая книга. }\end{array}$

В строке 23 пользователю нет необходимости использовать активирующую фразу, поскольку после окончания реплики голосового помощника прошло непродолжительное время, однако пользователь все равно начинает свою реплику с "Алиса", не столько "активируя" голосового помощника, сколько начиная новую тему. Мы видим, что даже в отсутствие необходимости «пробуждения" колонки пользователи обращаются к голосовому помощнику по имени, не полагаясь на поддержание связности разговора за счет удержания искусственным интеллектом предыдущего хода взаимодействия.

При этом, сколь бы уникальными ни были активирующие фразы, люди могут произносить или сами эти слова, или слова, похожие на них, в ходе обычных разговоров, тем самым активируя умную колонку тогда, как они этого не хотят. Это нечаянное начало разговора приводит к тому, что люди вынуждены прерывать взаимодействие (это одна из причин, по которой самое частое слово, используемое при взаимодействии с умными колонками,- “стоп» $\left.{ }^{7}\right)$. Чтобы избегать такого неуправляемого начала разговора, пользователи могут стараться не произносить ключевые слова, когда они, например, рассказывают кому-то о колонке в ее присутствии.

Другой аспект коммуникативных сбоев, с которыми сталкиваются пользователи умных колонок, заключается в том, что, если взаимодействие с колонкой включается в какую-то другую деятельность, сбой в этом взаимодействии может приводить к разрушению основной деятельности. Например, как показано в работе М. Поршерона и соавторов [Porcheron et al., 2018], если во время семейного ужина его участники пытаются заставить умную колонку поиграть с ними в игру или

\footnotetext{
7 По данным [Sciuto et al., 2018] и [Bentley et al., 2018].
} 
проиграть музыкальную композицию, но колонка их не понимает, это на какое-то время приводит к разрушению семейного ужина как специфической практики. Участники начинают фокусироваться на колонке, оставляя другие формы деятельности и переставая коммуницировать друг с другом.

Наконец, стоит отметить такую важную особенность одомашнивания искусственного интеллекта, как проявление вежливости. Речь идет о том, каким образом пользователи обращаются к умным колонкам, как они начинают и заканчивают разговор и реагируют на действия искусственного интеллекта. Помимо "официального" обращения по имени пользователи могут обращаться к колонке разными другими способами, в том числе теми, которые при взаимодействии с людьми сочли бы "невежливыми". Например, как показывают наши данные, в моменты коммуникативных сбоев пользователи могут называть колонку дурой. В то же время пользователи часто избегают привычных проявлений вежливости при взаимодействии с искусственным интеллектом. Это может быть связано с отсутствием ожидания, что искусственный интеллект будет "обижаться" не отсутствие "спасибо", "пожалуйста" и "до свидания", поскольку не способен понимать даже простые фразы. Но гораздо важнее, что искусственный интеллект воспринимается как компьютерная система, взаимодействие с которой носит более прагматический характер. Столкнувшись несколько раз с тем, что умная колонка неправильно понимает высказывание, пользователи избавляются от "непрагматических" компонентов взаимодействия вроде форм вежливости. Когда задача - произнести высказывание так, чтобы искусственный интеллект понял сказанное, это высказывание должно быть выстроено таким образом, чтобы максимально облегчить искусственному интеллекту задачу понимания: лучше говорить громко, медленно, с большими паузами и сообщать только то, что позволит добиться желаемого.

Но при рассмотрении роли вежливости во взаимодействии с умными колонками не следует забывать, что речь идет о специфическом типе домашних отношений, частью которых становится умная колонка. Как отмечалось выше, в доме колонка играет сервисную роль, "выполняя" просьбы пользователей. Эти сервисные отношения могут побуждать пользователей оценивать как свои реплики, так и реплики голосового помощника с точки зрения того, каким образом они отражают эти отношения. Рассмотрим следующий фрагмент:

04 понятно. алиса скажи какой компьютер

05 (.) лучше всего купить сейчас

$06 \quad(2.0)$

07 А ответ есть на выборовед точка ру (0.3)

08 читаю (.) приобрести хороший компьютер

09 (.) можно тремя способами. (0.2)

10 купить готовый (.) заказать сборку (.)

11 или сделать ее самому

$12(0.9)$

13 о очень информативно пасиба (0.6)

14 . х::: а как ты думае[шь-

15 А $[$ [поняла (.) исправлюсь

$16 \quad(0.5)$ 
$17 \Pi$ нет нет всё всё хорошㅇ всё хорошо

18 я (.) удовлетворен ответом (1.7)

Реплика Алисы в строке 15 становится для пользователя поводом для оценки того, как могла быть воспринята его реакция (строка 13) на ответ голосового помощника (строки 7-11). Судя по тому, как пользователь в строках 17 и 18 пытается исправить "неправильное" впечатление от своей реакции, он полагает, что Алиса сочла его реплику в строке 13 выражением неудовольствия полученным ответом. Независимо от того, насколько ироничной была фраза «очень информативно", пользователь при починке разговора привлекает контекст сервисных отношений, в соответствии с которыми его реплики выражают удовлетворенность предоставленной услугой (информацией). Подобная забота о том, чтобы "не обидеть" голосового помощника, свидетельствует, что контекст сервисных отношений способен "возвращать" определенные формы вежливого поведения во взаимодействие с голосовыми помощниками.

\section{Заключение}

Мы рассмотрели общие механизмы одомашнивания искусственного интеллекта, выделив ключевые аспекты домашних практик, частью которых становятся умные колонки. Мы показали, что умные колонки - это не просто новый объект, на который распространяются старые, привычные практики взаимодействия, а то, что трансформирует домашнюю социальность и побуждает изобретать новые формы действия. Дом, разумеется, не единственная среда, в которую будут проникать и уже проникают технологии искусственного интеллекта. Социальная организация каждой из этих сред накладывает свой отпечаток на то, как пользователи взаимодействуют с этими технологиями в своей повседневной жизни. Однако в той мере, в какой искусственный интеллект будет все больше предполагать использование голоса в качестве канала коммуникации с различными устройствами, между разными социальными обстановками будут обнаруживаться определенные сходства. Некоторые из них были отмечены в этой статье.

Как мы видели, одомашнивание искусственного интеллекта происходит не так, как предполагают разработчики. Социальные ситуации имеют собственную организацию, определяющую "естественность" взаимодействия с умными устройствами. Без учета этой организации, которую социологи выявляют, анализируя повседневные практики, технологии искусственного интеллекта будут в лучшем случае забавной игрушкой, а не полезной частью повседневной жизни. Социологи способны предоставить два типа описаний, которые могут лечь в основу разработки более социально ориентированных алгоритмов. С одной стороны, это детальный анализ различных повседневных культур (например, домашних ${ }^{8}$ ). С другой стороны, это детальный анализ практик взаимодействия с алгоритмами в естественных ситуациях. На наш взгляд, именно в этих двух направлениях должна развиваться социология искусственного интеллекта.

\footnotetext{
8 В качестве примера такого рода социологического анализа можно привести серию исследований группы британских социологов, занимавшихся изучением особенностей домашней среды в рамках проекта по разработке умного дома [Chamberlain, Crabtree, 2016; Crabtree, 2003; Crabtree, Rodden, 2004; Crabtree, Tolmie, 2016; Crabtree et al. 2015; Harper, 2003, 2011; Nilsson et al., 2019; Tolmie et al., 2008].
} 


\section{Список литературы (References)}

Блумер Г. Символический интеракционизм: перспектива и метод / пер. с англ. А. М. Корбута. М. : Элементарные формы, 2017.

Blumer H. (2017) Symbolic Interactionism: Perspective and Method. Moscow: Elementary Forms Press. (In Russ.)

Бурдье П. Практический смысл / пер. с фр. А. Т. Бикбова, К. Д. Вознесенской, С. Н. Зенкина, Н. А. Шматко; под ред. Н. А. Шматко. СПб. :Алетейя, 2001. Bourdieu P. (2001) Le sens pratique. Saint Petersburg: Aletheia. (In Russ.)

Ерофеева М.А. Проблематизация объекта в современной теоретической социологии // Социологические исследования. 2017. № 7. С. 13-23.

Erofeeva M. A. (2017) The Problem of the Object in Contemporary Social Theory. Sociological Studies. No. 7. P. 13-23. (In Russ.)

Латур Б. Пересборка социального: введение в акторно-сетевую теорию / пер. с англ. И. Полонской; под ред. С. Гавриленко. М. : Изд. дом Высшей школы экономики, 2014.

Latour B. (2014) Reassembling the Social: An Introduction to Actor-Network-Theory. Moscow: The Higher School of Economics Publishing House. (In Russ.)

Сакс Х., Щеглофф Э.А., Джефферсон Дж. Простейшая систематика организации очередности в разговоре / пер. с англ. А. М. Корбута // Социологическое обозрение. 2015. T. 14. № 1. С. 142-202. URL: https://sociologica.hse.ru/2015-141/147275640.html (дата обращения: 22.02.2021).

Sacks H., Schegloff E. A., Jefferson G. (2015) A Simplest Systematics for the Organization of Turn-Taking for Conversation. Russian Sociological Review. Vol. 14. No. 1. P. 142202. (In Russ.) URL: https://sociologica.hse.ru/2015-14-1/147275640.html (accessed: 22.02.2021).

Хокшилд А., Мачун Э. Вторая смена: работающие семьи и революция в доме / пер. с англ. И. Кушнаревой; под ред. А. Космарского. М. :Изд. дом Высшей школы экономики, 2020.

Hochschild A. R., Machung A. (2020) The Second Shift: Working Parents and the Revolution at Home. Moscow: The Higher School of Economics Publishing House. (In Russ.)

Alač M., Gluzman Y., Aflatoun T., Bari A., Jing B., Mozqueda G. (2020) Talking to a Toaster: How Everyday Interactions with Digital Voice Assistants Resist a Return to the Individual. Evental Aesthetics. Vol. 9. No. 1. P. 3-53. URL: https://eventalaesthetics.net/wp-content/uploads/2020/05/EAV9N1_2020_Alac_Toaster_3_53.pdf (accessed: 18.01.2021).

Aldrich F. K. (2003) Smart Homes: Past, Present and Future. In: Harper D. (ed.) Inside the Smart Home. London: Springer. P. 17-39. https://doi.org/10.1007/1-85233-854-7_2.

Bartlett S. N. (1997) Housing as a Factor in the Socialization of Children: A Critical Review of the Literature. Merrill-Palmer Quarterly. Vol. 43. No. 2. P. 169-198. 
Bentley F., LuVogt Ch., Silverman M., Wirasinghe R., White B., Lottridge D. (2018) Understanding the Long-Term Use of Smart Speaker Assistants. In: Proceedings of the ACM on Interactive, Mobile, Wearable and Ubiquitous Technologies. Art. No. 91. https://doi.org/10.1145/3264901.

Beneteau E., Richards O. K., Zhang M., Kientz J. A., Yip J., Hiniker A. (2019) Communication Breakdowns Between Families and Alexa. In: CHl'19: Proceedings of the 2019 CHI Conference on Human Factors in Computing Systems. Paper No. 243. https:// doi.org/10.1145/3290605.3300473.

Beneteau E., Guan Y., Richards O. K., Zhang M. R., Kientz J. A., Yip J., Hiniker A. (2020a) Assumptions Checked: How Families Learn About and Use the Echo Dot. In: Proceedings of the ACM on Interactive, Mobile, Wearable and Ubiquitous Technologies. Art. No. 3. https://doi.org/10.1145/3380993.

Beneteau E., Boone A., Wu Y., Kientz J. A., Yip J., Hiniker A. (2020b) Parenting With Alexa: Exploring the Introduction of Smart Speakers on Family Dynamics. In: $\mathrm{CHI} 20$ : Proceedings of the $2020 \mathrm{CHI}$ Conference on Human Factors in Computing Systems. https://doi.org/10.1145/3313831.3376344.

Cave S., Dihal K., Dillon S. (eds.) (2020) Al Narratives: A History of Imaginative Thinking About Intelligent Machines. Oxford: Oxford University Press. https://doi.org/10.1093/ oso/9780198846666.001.0001.

Chamberlain A., Crabtree A. (2016) Searching for Music: Understanding the Discovery, Acquisition, Processing and Organization of Music in a Domestic Setting for Design. Personal and Ubiquitous Computing. Vol. 20. No. 4. P. 559-571. https://doi.org/ 10.1007/s00779-016-0911-2.

Chambers D. (2020) Cultural Ideals of Home: The Social Dynamics of Domestic Space. London: Routledge.

Chevalier S. (1999) The French Two-Home Project: Materialization of Family Identity. In: Cieraad I. (ed.) At Home: An Anthropology of Domestic Space. Syracuse: Syracuse University Press. P. 83-94.

Crabtree A. (2003) The Social Organization of Communication in Domestic Settings. http://www.cs.nott.ac.uk/ pszaxc/work/IIEMCA03.pdf (accessed: 18.01.2021).

Crabtree A., Rodden T. (2004) Domestic Routines and Design for the Home. Computer Supported Cooperative Work. Vol. 13. No. 2. P. 191-220. https://doi.org/10.1023/b: cosu.0000045712.26840.a4.

Crabtree A., Rodden T., Tolmie P., Mortier R., Lodge T., Brundell P., Pantidi N. (2015) House Rules: The Collaborative Nature of Policy in Domestic Networks. Personal and Ubiquitous Computing. Vol. 19. No. 1. P. 203-215. https://doi.org/10.1007/ s00779-014-0771-6.

Crabtree A., Tolmie P. (2016) A Day in the Life of Things in the Home. In: CSCW'16: Proceedings of the 19th Conference on Computer Supported Cooperative Work (San 
Francisco (CA), USA, 27 February - 2 March 2016). New York: ACM Press. P. 17381750. https://doi.org/10.1145/2818048.2819954.

Csikszentmihalyi M., Rochberg-Halton E. (1981) The Meaning of Things: Domestic Symbols and the Self. Cambridge: Cambridge University Press.

Fischer J. E., Reeves S., Porcheron M., Sikveland O. (2019) Progressivity for Voice Interface Design. In: CUI'19: Proceedings of the 1st International Conference on Conversational User Interfaces. Art. No. 26. https://doi.org/10.1145/3342775.3342788.

Gilbert P. (2016) Classes, Gender, and Lifestyles in Domestic Space. Actes de la recherche en sciences sociales. Vol. 215. No. 5. P. 4-15.

Goffman E. (1961) Encounters: Two Studies in the Sociology of Interaction. Indianapolis: Bobbs-Merrill.

Grint K., Woolgar S. (1997) The Machine at Work: Technology, Work and Organization. Cambridge: Polity Press.

Harper D. (ed.) (2003) Inside the Smart Home. London: Springer.

Harper D. (ed.) (2011) The Connected Home: The Future of Domestic Life. London: Springer.

Hindus D. (1999) The Importance of Homes in Technology Research. In: Cooperative Buildings. Integrating Information, Organizations, and Architecture: Proceedings of the Second International Workshop, CoBuild'99 (Pittsburgh, PA, USA, October 1-2, 1999). Berlin: Springer. P. 199-207. https://doi.org/10.1007/10705432_18.

Lau J., Zimmerman B., Schaub F. (2018) Alexa, Are You Listening? Privacy Perceptions, Concerns and Privacy-Seeking Behaviors With Smart Speakers. In: Proceedings of the ACM on Human-Computer Interaction. Art. 102. https://doi.org/10.1145/3274371.

Malkin N., Deatrick J., Tong A., Wijesekera P., Egelman S., Wagner D. (2019) Privacy Attitudes of Smart Speaker Users. Proceedings on Privacy Enhancing Technologies. No. 4. P. 250-271. https://doi.org/10.2478/popets-2019-0068.

Mead G. H. (2015) Mind, Self, and Society. Chicago: University of Chicago Press.

Molotch H. (2011) Objects in Sociology. In: Clarke A. J. (ed.) Design Anthropology: Object Culture in the 21st Century. Vienna: Springer. P. 100-116. https://doi.org/10.1007/ 978-3-7091-0234-3_8.

Nevile M., Haddington P., Heinemann T., Rauniomaa M. (2014) On the Interactional Ecology of Objects. In: Nevile M., Haddington P., Heinemann T., Rauniomaa M. (eds.) Interacting with Objects: Language, Materiality, and Social Activity. Amsterdam: John Benjamins. P. 3-26. https://doi.org/10.1075/z.186.01int.

Nilsson T., Crabtree A., Fischer J., Koleva B. (2019) Breaching the Future: Understanding Human Challenges of Autonomous Systems for the Home. Personal and Ubiquitous Computing. Vol. 23. No. 2. P. 287-307. https://doi.org/10.1007/s00779019-01210-7. 
Oakley A. (2018) The Sociology of Housework. Bristol: Policy Press.

Phizacklea A., Wolkowitz C. (1995) Homeworking Women: Gender, Racism and Class at Work. London: Sage.

Pink S. (2004) Home Truths: Gender, Domestic Objects and Everyday Life. Oxford: Berg.

Pink S., Leder Mackley K., Moroşanu R., Mitchell V., Bhamra T. (2017) Making Homes: Ethnography and Design. London: Bloomsbury Academic. https://doi.org/10.4324/ 9781003085942.

Porcheron M., Fischer J. E., Reeves S., Sharples S. (2018) Voice Interfaces in Everyday Life. In: CHI'18: Proceedings of the $2018 \mathrm{CHI}$ Conference on Human Factors in Computing Systems (Montreal QC, Canada, April 21-26, 2018). Paper No. 640. https://doi.org/10.1145/3173574.3174214.

Reeves S. (2017) Some Conversational Challenges of Talking with Machines. In: Talking With Conversational Agents in Collaborative Action: Workshop at the 20th ACM Conference on Computer-Supported Cooperative Work and Social Computing (CSCW'17) (Portland, Oregon, USA, February 25-March 1, 2017). URL: https:// nottingham-repository.worktribe.com/output/844412/some-conversationalchallenges-of-talking-with-machines (accessed: 18.01.2020).

Riggins S. H. (1990) The Power of Things: The Role of Domestic Objects in the Presentation of Self. In: Riggins S. H. (ed.) Beyond Goffman: Studies on Communication, Institution, and Social Interaction. Berlin: Mouton de Gruyter. P. 341-368. https:// doi.org/10.1515/9783110847291.341.

Scicluna R. M. (2017) Home and Sexuality: The "Other" Side of the Kitchen. London: Palgrave Macmillan.

Sciuto A., Saini A., Forlizzi J., Hong J.I. (2018) “Hey Alexa, What's Up?”: Studies of InHome Conversational Agent Usage. In: DIS'18: Proceedings of the 2018 Designing Interactive Systems Conference (Hong Kong, China, June 2018). New York: ACM Press. P. 857-868. https://doi.org/10.1145/3196709.3196772.

Smart C. (2007) Personal Life. Cambridge: Polity Press.

Tolmie P., Crabtree A., Rodden T., Benford S. (2008) "Are You Watching This Film or What?": Interruption and the Juggling of Cohorts. In: CSCW'08. Proceedings of the ACM Conference on Computer Supported Cooperative Work (San Diego (CA), USA, 8-12 November 2008). New York: ACM Press. P. 257-266. https://doi.org/10.1145/ 1460563.1460605.

Voit A., Niess J., Eckerth C., Ernst M., Weingärtner H., Woźniak P.W. (2020) “It's Not a Romantic Relationship": Stories of Adoption and Abandonment of Smart Speakers at Home. In: Cauchard J., Löchtefeld M. (eds.) MUM 2020: 19th International Conference on Mobile and Ubiquitous Multimedia. New York: ACM. P. 71-82. https:// doi.org/10.1145/3428361.3428469. 